\title{
Windows Mobile Operating System Interface and Man-computer Interactive Design and Innovation
}

\author{
Liu Qingli,Lu Zhangping \\ School of art education \\ Jiangsu University \\ Beijing, China
}

\author{
He Canqun \\ School of art education \\ Jiangsu University \\ Beijing, China
}

\begin{abstract}
People are accustomed to using mobile Internet for browsing, chat, handling documents and emails to replace single GPS, MP3 and MP4. As the most portable personal information terminal, smart phone is undoubtedly the most intimate digital product. As different mobile phone operating systems have different interface styles and operation process inertias, there are different loyal users. On which system has the best man-computer interactive design? Windows Mobile is the software platform for portable devices such as pocket pc and Smartphone expands the familiar Windows desktop to personal devices. Windows Mobile has become a major mobile operating system. This system also has different interfaces. Through the analysis on three different operating interfaces of three mobile phone types to find out the advantages and disadvantages in man-computer interactive design. The result shows that windows Mobile operating system has the advantages in the man-computer interaction such as the interface has the similar PC Windows to facilitate the operation of people who are familiar with computer operation. However, windows Mobile system is complicated and difficult for people who are not familiar with computer operation. This is where Microsoft needs to be improved.
\end{abstract}

Keywords-Windows mobile, touch flo, man-computer interactive design

\section{INTRODUCTION}

People are accustomed to using mobile Internet for browsing, chat, handling documents and emails to replace single GPS, MP3 and MP4. As the most portable personal information terminal, smart phone is undoubtedly the most intimate digital product. Good user experience can gain more reputation and actual users for mobile phone operating system to enable a better interaction between smart phone and people, making more convenient and user-friendly operation process, less misuse and data loss [1].

Windows Mobile is the software platform for portable devices such as pocket pc and Smartphone. Windows Mobile expands the familiar Windows desktop to personal devices. It is the "mobile Windows" launched by Microsoft for handheld devices. Devices using the operating system of Windows Mobile mainly include PPC mobile phone, PDA and portable music players. There are three Windows Mobile operating systems, namely, Windows Mobile Standard, Windows Mobile Professional and Windows Mobile Classic.

As Microsoft Windows has a wide range of PC users, relatively speaking, Windows Mobile is the easiest smart phone operating system. All GUI (graphical interface) parts are the same as windows 98/XP, if you can use PC, you can directly operate the Windows Mobile smart phone. Currently, Microsoft's Windows Mobile system has been widely used in smart phones and handheld computers. Although the mobile phone market share is less than Symbian (Symbian operating system), it is accelerating to catch up. At present, the largest Windows Mobile cell phone manufacturers are: Taiwan HTC, Toshiba, HP, Mio, ASUS, Sony Ericsson, Samsung, LG, Motorola, Lenovo and Amoi, etc. This paper lists three Windows Mobile cell phones in different system versions with different man-computer characteristics, namely, HTC S1, Samsung i908E and HTC Diamond.

HTC S1

S1 uses Windows Mobile 6.1 system, and its screen resolution is only $320 \times 240$ pixels, which belongs to the standard configuration of Windows Mobile in one or two years before. Relative to the earlier Pocket PC2002 system, WM6.1 is much more gorgeous in color and button design, but its various menus and dialog boxes still keep the simple Microsoft line frame style.

The excellence of S1 is mainly manifested in its own Touch Flo plug: through the operation by figures on the screen, basic operations can be completed without pen. Touch Flo can also achieve special drag operation by user finger movements, for example, some software interface can be made from upward, whereas some user designated operation can be completed from left to right [3]. (Figure 3)

Of course, the advantages of Touch Flo are not just these, when we browse contacts, web pages or folders; we needed to conduct entry rolling by scroll bars in the past, whereas Touch Flo only need to slide your finger upward or downward in any part of the screen, and the entry will automatically scroll and slowly stop, as if having inertia. If the user sees the needed entry in the automatic scrolling process, he/she can stop scrolling by slightly touch the screen.

\section{A. Samsung SGH-i908E}

Samsung i908E is equipped with a 65,536-color TFT LCD screen with a viewing area of 3.2-inch, and a resolution of $400 \times 240$ pixels. Samsung Touch supports Touch Wiz touch technology, which can achieve dynamic icon drag and interface smooth switch. The left side of i908E screen has the similar tool bar as Vista and the user can freely drag the tools in the bar to the desktop for use; the screen will have a corresponding dynamic effect during icon drag, the tools 
dragged on the desktop can be automatically amplified to facilitate the user's operation. Despite Samsung has launched a number of Windows Mobile smart phone, the menu interface like i908E is the first. Use i908E touch control to freely carry out personalized decoration on i908E, in addition, functions can be performed in desktop home page, such as playing music, viewing pictures, adjusting time, alarm, etc; the reaction speed is also very fast; there is no need to enter menus at all levels can be described as one-step and the use of convenience can be imagined. In-depth user experience research makes i908E more user-friendly. (Figure 2)

\section{B. HTC Diamond}

HTC Diamond is equipped with a 2.8 -inch 65,536 color TFT touch screen with a resolution of $480 \times 800$ pixels. This smart phone uses new Windows Mobile 6.1 Professional operational system, with built-in Touch Flo 3D interface. Touch Flo 3D interface integrates the standby mode, common contacts, information, e-mail, web browser, photo and video, music player, weather forecasting, phone setting and procedure assembly, etc; the functional project switch can be achieved by sliding thumb in the icon bar. It adds more 3D animation effects; the display of common contact and weather forecast is the best description. (Figure 1) Moreover, the second generation of Diamond has also been launched, and there is no difference with the first generation. With the increase in screen height, the screen resolution has changed into $480 \times 800$ pixels, and enriched video content. What worth mentioning is Diamond series have built-in gravity sensor system. This system can adjust screen content for horizontal screen viewing according to the phone tilt angle, which is particular convenient in viewing photos and enter text by using the virtual keyboard by both hands.

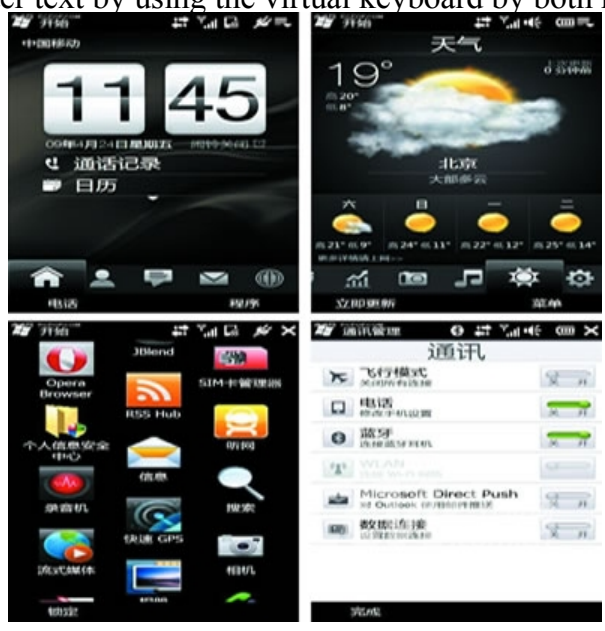

Figure 1. 3d flo touch interface

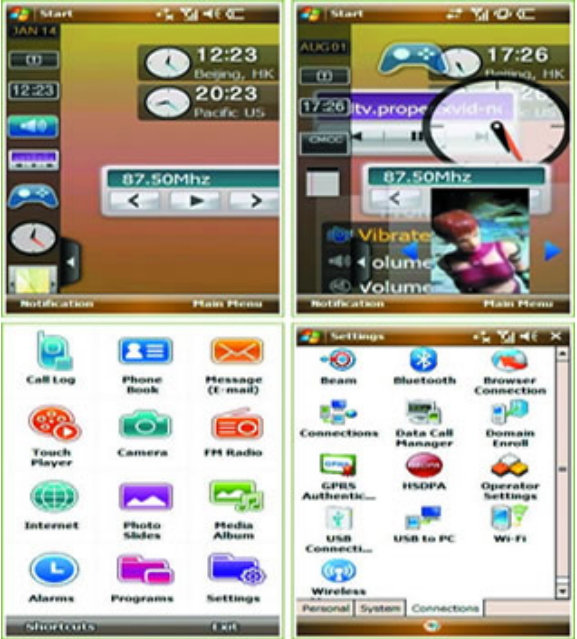

Figure 2. Wiz touch interface

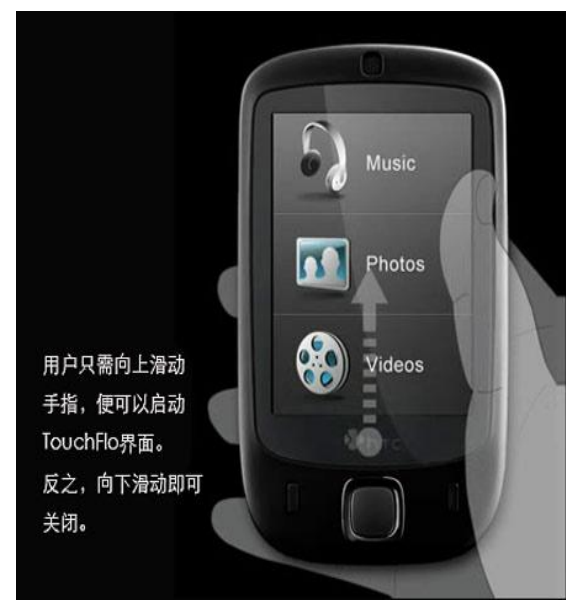

Figure 3. Flo touch interface

\section{Windows Mobile Phone Operating System INTERFACE INNOVATION}

The use of personal mobile communication terminal cannot do without the impact of user interaction scenario conditions. What is the user interaction mode? The concept interpretation is: it is a description of the implementation process of user interaction task and using environment, which completes the mission objectives in the form of information input and output. In the process, the user is required to accurately enter the command, information, etc through the finger or other means. In different environment, the user's input accuracy will have large differences, such as under the circumstances of static and slow states, walking and car shaking, etc, the information input accuracy and displayed cognitive efficiency will rapidly decline with the environmental interference [2]. It can be seen that the existing mobile phone scene mode definitions mainly conduct information output and cognitive reminder on users in terms of interface display and by using user-aware functions such as sound, light and vibration, rather than 
granting some consideration and innovation to the information entry convenience and the validity of integrated interactive aspects of interface display. How to break the limitations of conventional interactions to make multiple channels of interactions (that is, users can choose appropriate means of interactions according to environment changes), which will be the trend of interactive means of personal hand-held communication terminal [4].

For the interface of Windows Mobile phone operating system, its man-computer interface design has been developed and optimized to a very advanced stage, while users will come across the following obstacles in using the smart phone of Windows Mobile phone operating system interface: 1. Disordered and complicated system interface, difficult to use. 2. In cell phone standby, the keyboard is always forgotten to open. As it is a touch screen mobile phone, it often leads to misuse. 3. In message text, the users of Windows Mobile smart phones often need to complete text input by both hands, so it is quite in convenient when users are in the bus, subway or walking state. On these operation obstacles, we can make improvement and innovation by the following approaches:

\section{A. Adjust standby interface design, increase simple shortcuts}

Design simple and concise Windows Mobile phone operating system standby interface design, only retain the date and time, and add the call shortcut. This design can not only reduce the memory of Windows Mobile and can reduce the standby power consumption at the same time, and more facilitate the operation of new users. At the same time, it is necessary to add a shortcut to the frequently used software manager. In using Windows Mobile smart phone, users often need to click "start"-"program" before entering the common software operating interface. If add a software manager in the standby mode, the user clicks can be reduced to facilitate the directly access to commonly used software such as Office, Internet Explorer and Media Player.

\section{B. Add keyboard lock shortcut in the Windows Mobile phone standby interface}

In system setting, add auto-lock function. In the operation of Windows Mobile phone, if no new operation occurs within a certain time range, the system will automatically lock. To some extent, mis-operation can be avoided when the user forgets to lock the keyboard.

\section{In Windows Mobile phone text input, add standard keyboard text input method for the Chinese users}

Chinese users mainly use Pinyin and stroke input methods in the selection of text message input, while in using Windows Mobile phones, the previous version only has handwriting input; despite the new generation Windows Mobile phone add Pinyin input, it is full keyboard input. It is hard to imagine how many misuses will appear in text message in the 26-letter standard keyboard in a small screen. Therefore, the Microsoft should consider adding the standard keyboard and association input in the design of Windows Mobile phone message input interface for Chinese users. If so, Chinese Window Mobile phone users can send text messages with one hand as easy as the cell phones with standard keyboard.

\section{CONCLUSIONS}

after the above analysis, the Windows Mobile operating system has the following advantages in the man-computer interaction; 1 . the interface has the similar PC Windows to facilitate the operation of people who are familiar with computer operation. 2. Rich preload software, with built-in Office Word, Excel, Power Pont, which can be browsed or even edited, with built-in Internet Explorer and Media Player. 3. Very convenient PC synchronization, fully compatible with Outlook, Office Word and Excel, etc. 4. Powerful multimedia; can play almost any popular formats of audio and video files with the third-party software. 5. Touch type operation, powerful, simple and practical. 6. Extremely rich third-party software, particularly the dictionary, satellite navigation software can be run. But at the same time, Windows Mobile system is complicated and difficult for people who are not familiar with computer operation. This is where Microsoft needs to be improved, with the release of Microsoft's new operating system Windows Phone 7, the new generation operating system should be easier to use and more user-friendly.

\section{REFERENCES}

[1] Zhou Rui. On the Development Trend of Mobile Phone Interface Design Interaction Approach, Journal of Nanjing Arts Institute [J], 2007, PP: 129-133

[2] Heim. Harmonious Interaction the Basis of Interaction Design [M] translated by Li Xueqing, Beijing, Electronic Industry Press, 2008

[3] Li Yinan. On the Main Characteristics of Good Interaction Design [J] Packaging Engineering, 2008, PP: 162-164

[4] Cui Tianjian Industrial Product Design Theory and Techniques [M] Nanjing, Southeast University Press, 2005 